DOI: 10.1007/s00350-016-4318-x

\section{Anmerkung zu OLG Karlsruhe, Urt. v. 12.11.2015 - 9 U 78/11 (LG Konstanz)}

\section{Max Middendorf}

Der Senat hat die Voraussetzungen für die sofortige Unterbringung geprüft und unter zwei Aspekten verneint. Es mangelte vorliegend schon an dem ersten Kriterium, nämlich der drohenden Eigen- oder Fremdgefährdung. In diesem Zusammenhang ist zunächst auf eine Selbstverständlichkeit rekurriert worden, nämlich dass ärztliche Zeugnisse - wie jede ärztlicherseits geschuldete Handlung - den fachlich anerkannten Standards entsprechen müssen. An dieser Stelle gelten für Amtsträger über Art. 34 GG/\$839 BGB die gleichen Maßstäbe wie für den privatrechtlich Handelnden (\$S630a Abs. 2, 823 Abs. 1 BGB). Bereits daran fehlte es hier nach der Aktenlage. Es fehlten in den ärztlichen Aufzeichnungen hinreichende Anhaltspunkte dafür, die die Gefährdungsprognose hätten stützen können.

Der Senat hat - sachverständig beraten - ganz entscheidend darauf abgestellt, dass es ein elementarer Standard ist, die konkreten Anknüpfungstatsachen darzustellen, aus denen sich die Gefährdungsprognose ergeben soll. Diese Anknüpfungstatsachen lagen hier aber entweder nicht vor oder sie waren nicht dokumentiert und konnten im Ergebnis nicht belegt werden (vgl. insoweit $\$ 630 \mathrm{~h}$ Abs. 3 BGB), so dass ein Verstoß gegen die Pflichten bei der Erstellung eines ärztlichen Zeugnisses angenommen wurde. Allein die u. U. sogar zutreffende Diagnose einer Psychose rechtfertigte nicht die zwingend erforderliche Gefährdungsprognose.

Hinzu kam, dass vorliegend eine sofortige Unterbringung beantragt worden war. Auch unter diesem Aspekt war der Antrag rechtswidrig, denn die antragstellenden Ärzte hatten keinerlei Umstände angeführt, die eine besondere Dringlichkeit der Maßnahme transparent gemacht hätten.

Die Ärzte konnten sich nicht auf eine Unterbrechung des Zurechnungszusammenhanges zurückziehen. Das Argument, die eigentliche freiheitsentziehende Maßnahme rühre vom Vormundschaftsgericht her, verfing nicht. Der Senat hat zutreffend deutlich gemacht, dass ein Antrag von einer ,anerkannten Einrichtung“ i. S. des UBG BW - hier: des Zentrums für Psychiatrie - conditio sine qua non für das Eingreifen des Vormundschaftsgerichts war. Zudem war das Vormundschaftsgericht seinerseits von der ärztlichen Expertise in den angegriffenen Zeugnissen abhängig, so dass die Kausalität der rechtswidrigen Handlung für die Freiheitsentziehung und die Zwangsmedikation richtigerweise bejaht wurde.

Schließlich verdient die Höhe des zuerkannten Schmerzensgeldbetrages Beachtung. Besondere körperliche Schäden waren - soweit ersichtlich - trotz Zwangsmedikation nicht eingetreten. Der Senat hat sich hier jedoch, ohne dies näher zu benennen, ersichtlich von der Bedeutung der Grundrechte der körperlichen Fortbewegungsfreiheit und des Allgemeinen Persönlichkeitsrechts leiten lassen (jeweils Art. 2 Abs. 1 GG). Die Genugtuungsfunktion spielt zumindest im klassischen Bereich der fehlerhaften ärztlichen Behandlung für die Schmerzensgeldsbemessung grundsätzlich keine Rolle ${ }^{1}$ mehr, hier hat das Gericht jedoch ausdrücklich auch auf die Schwere des Verschuldens abgestellt. Zudem wurden die mit der Maßnahme verbundene Demütigung und die potentiell stigmatisierende Wirkung betont.

Rechtsanwalt Dr. iur. Max Middendorf,

Fachanwalt für Medizinrecht, Lehrbeauftragter an der

WWU Münster, Bergmann und Partner,

Josef-Schlichter-Allee 38, 59063 Hamm, Deutschland
Zusammenfassend bleibt festzuhalten, dass die Entscheidung Zustimmung verdient. Der Senat hat zu Recht hohe Anforderungen an den Nachweis der Voraussetzungen für die Unterbringung gestellt. Die Entscheidung ist bereits an dieser Stelle vom Blick auf die Grundrechte geprägt, obwohl diese in den Gründen keine nähere Erwähnung erfahren. Diese Linie setzt sich auch in den Rechtsfolgen, namentlich der vergleichsweise hohen Schmerzensgeldbemessung fort. Vergleich man den zuerkannten Betrag mit solchen Beträgen, die zum Teil für erhebliche Dauerschäden zuerkannt werden, so scheint hier neben der Ausgleichsfunktion ein präventives Momentum eine Rolle gespielt zu haben, wenngleich dies vom Senat nicht explizit ausgesprochen wurde. Richtig ist es schließlich auch, dass bei der Bemessung der Höhe des Ersatzanspruches der Verweis auf die strafrechtlichen Entschädigungsregelungen verworfen wurde, da die Vergleichbarkeit mit der verschuldensabhängigen Amtshaftung schon unter systematischen Gesichtspunkten verfehlt wäre.

1) Vgl. etwa OLG Düsseldorf, Urt. v. 28.9.2002 - 8 U 190/01 -, NJW-RR 2003, 87 = VersR 2004, 120.

\section{Einsichtnahme in die Patientenakte}

\section{BGB $\S 630 \mathrm{~g}$}

Zur Frage der Erforderlichkeit einer Vollmachtsvorlage bei anwaltlicher Geltendmachung des Rechts zur Einsichtnahme in die Behandlungsdokumentation. (Leitsatz des Bearbeiters)

LG Stuttgart, Beschl. v. 9.12.2015 - 19 T 488/15 (AG Ludwigsburg)

Problemstellung: Der Patient hat (auch) außerhalb eines Rechtsstreits ein Recht auf Einsicht in die Behandlungsdokumentation (vgl. nur Katzenmeier, in: Laufs/Katzenmeier/Lipp, Arztrecht, 7. Aufl. 2015, Kap. IX, Rdnrn. 55 ff.; zum im Detail uneinheitlich gehandhabten und nicht hinreichend geklärten prozessualen Einsichtsrecht vgl. etwa OLG Karlsruhe, NJW-RR 2013, 312; OLG Hamm, BeckRS 2007, 05080; s. auch Katzenmeier, a.a.O., Kap. IX, Rdnr. 64). Diese Selbstverständlichkeit ist seit dem Inkrafttreten des Gesetzes zur Verbesserung der Rechte von Patientinnen und Patienten am 26.2.2013 für den vertraglichen Bereich ausdrücklich in $\$ 630 \mathrm{~g}$ Abs. 1 BGB geregelt. Durch die gesetzliche Regelung wurde das Einsichtsrecht des Patienten ausgeweitet, da es sich nach der Intention des Gesetzgebers auf die vollständige, ihn betreffende Patientenakte erstreckt und nur bei Entgegenstehen erheblicher therapeutischer Gründe oder sonstiger erheblicher Rechte Dritter eine Ausnahme vorsieht (vgl. nur Deutsch/Spickhoff, Medizinrecht, 7. Aufl. 2014, Rdnr. 922; Frahm/Nixdorf/Walter, Arzthaftungsrecht, 5. Aufl. 2013, Rdnr. 153). Mag die Ausgestaltung der Vorschrift zur Klarstellung einiger in der Vergangenheit diskutierter rechtlicher Aspekte etwa des Einsichtsorts und des Rechts auf Kopien (eigene oder gefertigte gegen Kostentragung) - beigetragen haben (vgl. zum Ganzen etwa Kensy, MedR 2013, 767), stellen sich in der Praxis doch immer wieder offene Probleme. Zu denken ist nur an die Frage, was ,unverzüglich“ i. S. des $₫ 630 \mathrm{~g}$ Abs. 1 BGB bedeutet oder welche

Eingesandt von Rechtsanwalt Tobias Rist, Stuttgart;

bearbeitet von RiOLG Dr. iur. Alexander Walter,

OLG Koblenz,

Stresemannstraße 1, 56068 Koblenz, Deutschland 